\title{
FIGO Stage IIB
}

National Cancer Institute

\section{Source}

National Cancer Institute. FlGO Stage IIB. NCI Thesaurus. Code C96254.

A FIGO stage term that applies to gynecologic cancers. For cervical cancer, it refers to cancer that invades beyond the cervix, but not to the pelvic wall or lower third of the vagina and there is parametrial invasion; for endometrial cancer, in the older FIGO classification stage scheme, it referred to cervical stromal invasion. Stage IIB for endometrial cancer has been eliminated in the most recent (2010) FIGO classification stage scheme. 